\title{
Über Bewertungen
}

\section{Eberhard Wolff}

Prof. Dr. rer. soc., Redaktor Kultur, Geschichte, Gesellschaft

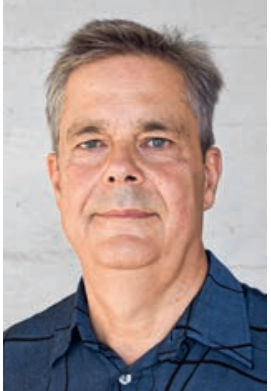

Literatur

1 Meienberg O. Arzt-Entwertung im Internet. Schweiz Ärzteztg. 2018;99(34):1114-7.

2 https://www.konsumentenschutz.ch/ medienmitteilungen/2003/03/allianzguide-sante/

Barnikol M, Sojer R,

Röthlisberger F. Rechtliche Aspekte zu Bewertungen im Internet. Schweiz Ärzteztg. 2019;100(19):634-6.
Als ich neulich im Web nach einer früheren Bekannten forschte, fand ich nur ihren älteren Bruder, Zahnarzt in Deutschland. Seine Praxis hat keine Website, trotzdem gibt es online ein paar Bewertungen über ihn. Eine von ihnen meint, er sei grobschlächtig wie ein Pferdedoktor. Eine andere lobt ihn wegen seiner Vorsicht.

Nicht nur wegen der möglichen Persönlichkeitsverletzungen wurden und werden in der Ärzteschaft grundsätzliche Vorbehalte gegen Online-Bewertungen ihrer Tätigkeit geäussert: Können komplexe ärztliche Behandlungen überhaupt angemessen durch Patientinnen und Patienten bewertet werden? Bewertungen von Ärzten passten auch nicht neben «Pizza-Kuriere, Shopping Centers, Autowaschanlagen» [1].

Vor allem seit dem 19. Jahrhundert zählte es zu den Grundpfeilern ärztlicher Standespolitik, den herausgehobenen, möglichst autonomen Status des Arztberufs zu verteidigen. Das heisst nicht zuletzt, sich als Stand selbst zu kontrollieren. Bewertungen von aussen passen da schlecht. Vielleicht war das einer der Gründe, warum vor einigen Jahren ein geplanter Guide Santé der Organisationen von Patientinnen und Patienten, von Konsumentinnen und Konsumenten ohne die Mitarbeit der schweizerischen Ärzteschaft letztlich nicht zustande kam [2]. Ärztebewertungen vonseiten der Klientel hat es allerdings immer gegeben. Sie liefen auch ohne Internet, zumeist als persönliche Mund-zu-Mund-Empfehlungen oder als allgemeine Medizinkritik. Online läuft das Bewerten heute «heisser»: häufiger, öffentlicher, schneller und emotionaler. Ähnlich bei den Journalistinnen und Journalisten. Sie haben immer schon Leserbriefe auf ihre Artikel erhalten. Heute kämpfen manche Zeitschriften damit, die Kommentarfunktion im Zaum zu halten. Ärztinnen und Ärzte in der Schweiz arrangieren sich jetzt aber zunehmend grundsätzlich mit den Online-Bewertungen. Die FMH wird demnächst differenzierte Empfehlungen veröffentlichen [3].

Ob man sie bekämpft oder mit ihnen arbeitet: Web-Bewertungen - auch von Ärztinnen, Ärzten oder ganzen Praxen - sind eine Tatsache, die sich nicht einfach aus der Welt schaffen lässt. Sie sind nicht nur als Kinder des digitalen Zeitalters allgegenwärtig, sondern auch als Teil eines generellen Trends zu Evaluation, Assessment, Rating, Outcome oder Feedback, auch und gerade in der Medizin. Im Internet werden sogar Bewertungen bewertet.

Damit gehen Extremphänomene wie «Cybermobbing» und «Shitstorms» einher. Oder das Menschen-Rating, wie es in China zurzeit vorbereitet und auf Netflix in den «Black-Mirror»-Dystopien bitterbös in Szene gesetzt wird. Andere kritisieren, dass die neoliberale Bewertungskultur Menschen ständig zu noch höheren Leistungen antreibe.

Bewertungen sind aber auch Teil eines unspektakulären Alltags. Sie bieten ein Stück Orientierung. Neue digitale Privatanbieter-Märkte wie eBay, Airbnb oder Uber funktionieren nur mit einem System ständiger gegenseitiger Bewertungen. Dies schafft eine Art vertrauensbildender Geschäftsgrundlage. Und wer von Ihnen hat noch nie ein Hotel auch anhand dessen Kundenbewertungen ausgesucht?

Mit der Masse wandelt sich der Stellenwert von Bewertungen. Wie lesen Sie selber Hotelbewertungen z.B. bei TripAdvisor oder HolidayCheck? Glauben Sie wirklich jedes Wort? Schreckt Sie bereits ein einzelner «Verriss» ab? Denken Sie bei besonders guten Bewertungen auch, dass sie gekauft sein könnten? Wer häufig Bewertungen und Evaluationen gleich welcher Art liest, entwickelt daraus eine skeptische Distanz und ein Gespür für den ungefähren Gesamteindruck nicht nur von Hotels. Die Methode dahinter dürfte dem diagnostischen Gespür erfahrener Ärztinnen und Ärzte bei der Anamnese jenseits einzelner Aussagen nicht unähnlich sein.

Eben erst hat eine Werbekampagne der Firma digitec die ganze Schweiz mit schrägen oder lustigen Kundenrezensionen vollplakatiert. Augenzwinkernd machen sie sich über die Bewertungen lustig. Es seien «ehrliche Meinungen». Sie sagen nicht: «zutreffende». Denn Bewertungen sind nie "objektiv». Bei digitec wissen sie ganz genau, dass es nicht auf den Inhalt der einzelnen Bewertung ankommt. Es ist die Stimmung einer "ehrlichen» Community, die das Grundvertrauen und positive Image schaffen soll.

Der Medizinbereich funktioniert sicher nicht wie TripAdvisor. Online-Bewertungen werden in unserem Gesundheitswesen kaum je eine zentrale Rolle spielen. Wer aber sieht, wie Bewertungen anderswo funktionieren, weiss sie auch in der Medizin in ihrer Vielfältigkeit besser einzuschätzen.

Die Praxis des genannten Zahnarztes wird weiterlaufen, trotz des Ärgers, dass ihn jemand online einen Pferdedoktor schimpft.

PS: Wie fanden Sie diesen Artikel? O genial, O blabla, 0 hatdersienochalle, 0 wasweissich. 